\title{
Combining targeted metabolite analyses and transcriptomics to reveal the specific chemical composition and associated genes in the incompatible soybean variety PI437654 infected with soybean cyst nematode HG1.2.3.5.7
}

Xue Shi ${ }^{1 \dagger}$, Qiansi Chen ${ }^{2 \dagger}$, Shiming Liu', Jiajun Wang ${ }^{3}$, Deliang Peng ${ }^{1 *}$ and Lingan Kong ${ }^{1 *}$

\begin{abstract}
Background: Soybean cyst nematode, Heterodera glycines, is one of the most devastating pathogens of soybean and causes severe annual yield losses worldwide. Different soybean varieties exhibit different responses to $H$. glycines infection at various levels, such as the genomic, transcriptional, proteomic and metabolomic levels. However, there have not yet been any reports of the differential responses of incompatible and compatible soybean varieties infected with $\mathrm{H}$. glycines based on combined metabolomic and transcriptomic analyses.

Results: In this study, the incompatible soybean variety PI437654 and three compatible soybean varieties, Williams 82, Zhonghuang 13 and Hefeng 47, were used to clarify the differences in metabolites and transcriptomics before and after the infection with HG1.2.3.5.7. A local metabolite-calibrated database was used to identify potentially differential metabolites, and the differences in metabolites and metabolic pathways were compared between the incompatible and compatible soybean varieties after inoculation with HG1.2.3.5.7. In total, 37 differential metabolites and 20 KEGG metabolic pathways were identified, which were divided into three categories: metabolites/pathways overlapped in the incompatible and compatible soybeans, and metabolites/pathways specific to either the incompatible or compatible soybean varieties. Twelve differential metabolites were found to be involved in predicted KEGG metabolite pathways. Moreover, 14 specific differential metabolites (such as significantly upregulated nicotine and down-regulated D-aspartic acid) and their associated KEGG pathways (such as the tropane, piperidine and pyridine alkaloid biosynthesis, alanine, aspartate and glutamate metabolism, sphingolipid metabolism and arginine biosynthesis) were significantly altered and abundantly enriched in the incompatible (Continued on next page)
\end{abstract}

\footnotetext{
* Correspondence: pengdeliang@caas.cn; konglingan@caas.cn

${ }^{+}$Xue Shi and Qiansi Chen contributed equally to this work.

'State Key Laboratory for Biology of Plant Diseases and Insect Pests, Institute

of Plant Protection, Chinese Academy of Agricultural Sciences, Beijing

100193, China

Full list of author information is available at the end of the article
}

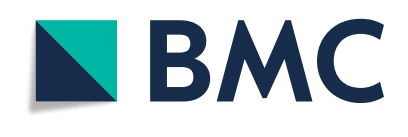

(- The Author(s). 2021 Open Access This article is licensed under a Creative Commons Attribution 4.0 International License, which permits use, sharing, adaptation, distribution and reproduction in any medium or format, as long as you give appropriate credit to the original author(s) and the source, provide a link to the Creative Commons licence, and indicate if changes were made. The images or other third party material in this article are included in the article's Creative Commons licence, unless indicated otherwise in a credit line to the material. If material is not included in the article's Creative Commons licence and your intended use is not permitted by statutory regulation or exceeds the permitted use, you will need to obtain permission directly from the copyright holder. To view a copy of this licence, visit http://creativecommons.org/licenses/by/4.0/. The Creative Commons Public Domain Dedication waiver (http://creativecommons.org/publicdomain/zero/1.0/) applies to the data made available in this article, unless otherwise stated in a credit line to the data. 
(Continued from previous page)

soybean variety PI437654, and likely played pivotal roles in defending against HG1.2.3.5.7 infection. Three key metabolites (N-acetyltranexamic acid, nicotine and D,L-tryptophan) found to be significantly up-regulated in the incompatible soybean variety PI437654 infected by HG1.2.3.5.7 were classified into two types and used for combined analyses with the transcriptomic expression profiling. Associated genes were predicted, along with the likely corresponding biological processes, cellular components, molecular functions and pathways.

Conclusions: Our results not only identified potential novel metabolites and associated genes involved in the incompatible response of PI437654 to soybean cyst nematode HG1.2.3.5.7, but also provided new insights into the interactions between soybeans and soybean cyst nematodes.

Keywords: Incompatible and compatible soybean varieties, Soybean cyst nematode, Metabolomic analyses, Transcriptomics, Combination analyses

\section{Background}

Soybean cyst nematode (SCN), Heterodera glycines, is one of the most devastating pathogens of soybean and causes a large annual yield losses worldwide [1-4]. SCN is a typical obligate endoparasitic nematode [3]. The juveniles of SCN pierce the soybean root with a spear-like feeding structure called a stylet to penetrate the soybean root $[3,5]$. They then invade soybean roots and migrate to the vascular bundles, where they establish a complex feeding site known as a syncytium [6, 7]. The formation and maintenance of the syncytium lead to dramatic changes in the internal root structure, affect the root system and cause damage [3]. Additionally, soybean roots produce many defense-related compounds, such as reactive oxygen species, and activate hormone signaling pathways in response to nematode infection, and great changes in metabolites are observed in soybean roots upon SCN infection $[8,9]$.

Recently, the use of "omics" technology to obtain biological data has surged, and the most commonly used omics approaches are genomics, transcriptomics, proteomics and metabolomics [10-12]. There are numerous research reports addressing soybean-SCN interactions on the basis of genomic and transcriptomic analyses [4, 1320]. The genomes of SCN and many soybean varieties have been evaluated to further investigate their biology, characteristics and potential interactions [4, 19, 21-24]. Many candidate soybean resistance loci and/or genes have been identified based on the genome-wide association studies (GWAS) [13, 18, 20]. Moreover, many studies on the expression profiling of different plant tissues, such as the whole root samples and the syncytium structure, have been carried out in compatible and incompatible soybean varieties infected with $\mathrm{SCN}$, and many differentially expressed genes (DEGs) and related KEGG pathways have been predicted $[14,15,17,25,26]$.

Metabolomics is used to characterize metabolic patterns and further study the functions and significance of key differential metabolites that play important roles in biological and medical fields [27-29]. Plant metabolomics has gained increasing attention and has become a mature method for studying plant responses to both biotic and abiotic stresses. Therefore, plant metabolomics is considered as an indispensable tool in the study of plant-pathogen interactions [30]. Plants respond to pathogen infection by producing defenserelated metabolites, especially the plant secondary metabolites, which play important roles in plant resistance [31]. Metabolomics can provide technical support for the identification of plant metabolites, which are potential sources of new compounds for nematode control [32]. Research on plant nematodes has become increasingly abundant, especially work addressing root-knot nematodes. Eloh et al. performed metabolomic analysis of root-knot nematodes treated with maleimide and proved that maleimide could be used as a new potential nematicide [33]. In addition, they studied the levels of metabolites in tomato plants after root-knot nematode infection, and found that tomato roots showed changes in biochemical pathways in response to root-knot nematode infection [33]. Recently, Kantor et al. investigated the metabolite profiles of both incompatible and compatible watermelon accessions with root-knot nematode infection and found that the roots of incompatible wild watermelon accessions were rich in metabolic compounds that had a nematicidal effect but these compounds showed decreased levels in commercial watermelon cultivars [31]. However, there have been a few studies on soybean-SCN interactions based on proteomic and metabolomic analyses [34, 35]. Differential proteins and metabolites between incompatible and compatible soybean roots were identified with the traditional approaches more than ten years ago [34]. Recently, Kang et al. studied the effect of Bacillus simplex strain Sneb545 on soybean secondary metabolites under SCN infection by combining advanced and highthroughput approaches of transcriptomic and metabolomic analyses and found that Sneb545-treated soybeans showed higher concentrations of various nematicidal metabolites [35]. 
To date, there have been no reports on the differences in metabolic pathways between incompatible and compatible soybean varieties infected with soybean cyst nematodes. In this study, an incompatible soybean variety PI437654 [36-38] and three compatible soybean varieties, Williams 82 (WM82), Zhonghuang 13 (ZH13) and Hefeng 47 (HF47), were selected as the research materials, and LC/MS full-scan detection technology was used to investigate the differences in metabolites and metabolic pathways between the incompatible and compatible soybean varieties after inoculation with HG1.2.3.5.7. Metabolites overlapped between the incompatible and compatible soybean varieties, differential metabolites specific to the incompatible or compatible soybean varieties and the potential KEGG metabolic pathways of these metabolites were identified. Moreover, the genes associated with the significantly up-regulated differential metabolites were predicted, and the associated genes were classified into two types. One type of the genes were associated with significantly up-regulated differential metabolites specific to the incompatible soybean variety PI437654, and the other type of the genes were associated with the overlapping differential metabolites that were significantly up-regulated in the incompatible soybean variety PI437654 but simultaneously significantly down-regulated in the three compatible soybean varieties. The results not only identified potential novel metabolites and associated genes involved in the incompatible response of PI437654 to soybean cyst nematode HG1.2.3.5.7, but also provided new insights into the interaction between soybeans and soybean cyst nematodes.

\section{Results}

Differentiation of the metabolites between incompatible and compatible soybean varieties under infection with HG1.2.3.5.7

To investigate the differential root metabolites between the incompatible and compatible soybean varieties, the root samples of the incompatible soybean variety PI437654 and three compatible soybean varieties (WM82, ZH13 and HF47) (Fig. S1, Table S1) infected with SCN HG1.2.3.5.7 ('_SCN') at 8 dpi were collected, and their corresponding control root samples inoculated with water ('_0') were also collected, respectively. All the root samples were subjected to metabolomic analyses. In the PCA chart of incompatible soybean, all six replicate samples of PI437654_SCN were clustered together, and all six replicate samples of PI437654_0 were also clustered together (Fig. 1a and S2). Moreover, the PI437654 SCN samples were clearly separated from those of PI437654_0 (Fig. 1a and S2), which suggested that there were significant changes in metabolites in the incompatible soybean variety PI437654 after inoculation with
HG1.2.3.5.7. The PLS-DA results were similar to those of the PCA, and the samples of PI437654_SCN were also clearly separated from those of PI437654_0 (Fig. 1b and S2). The PCA and PLS-DA results showed that all three compatible soybean varieties, WM82_SCN, ZH13_SCN and HF47_SCN, were dramatically separated from WM82_0, ZH13_0, and HF47_0, respectively (Fig. 1a, b and S2), which indicated that the inoculation of HG1 2.3.5.7 caused obvious changes in the root metabolites of the three compatible soybean varieties. Furthermore, according to the cumulative interpretation rate of the model for the incompatible and compatible soybean varieties (Fig. 1c, Table S2), both the $\mathrm{R}^{2}$ and $\mathrm{Q}^{2}$ values were close to 1 , clearly indicating that the predictive power and quality of the two groups of models were suitable for the subsequent experiments. These results indicated that the inoculation of HG1.2.3.5.7 caused significant metabolic changes in both the incompatible soybean variety PI437654 and the three compatible soybean varieties.

\section{Identification of differential metabolites in incompatible and compatible soybean varieties}

In total, 19 significantly differential metabolites were screened from the incompatible soybean variety PI437654 infected with HG1.2.3.5.7 at 8 dpi compared to that in the corresponding control of PI437654 inoculated with water (designated PI437654_SCN vs PI437654_0), among which seven metabolites were upregulated, and 12 metabolites were down-regulated (Table 1). In the compatible soybean WM82_SCN vs WM82_0, 17 obviously differential metabolites were identified, including three up-regulated and 14 downregulated metabolites (Table 1). Similarly, there were 12 dramatically differential metabolites in ZH13_SCN vs ZH13_0, all of which were down-regulated (Table 1). There were 17 dramatically differential metabolites in HF47_SCN vs HF47_0, including three up-regulated metabolites and 14 down-regulated metabolites (Table 1). The differential metabolites among the four soybean varieties exhibited different profiles, which were mainly divided into the following three categories.

(1) Overlapping differential metabolites between the incompatible soybean variety PI437654 and the three compatible soybean varieties. There were four overlapping differential metabolites (Table 1, Fig. S3), including D-leucine, D,L-tryptophan, 16hydroxyhexadecanoic acid and linolenic acid, which were amino acids and fatty acids. Linolenic acid was simultaneously dramatically down-regulated in the incompatible soybean variety PI437654_SCN vs PI437654_0 and the three compatible soybean varieties. D-leucine was dramatically down-regulated in 


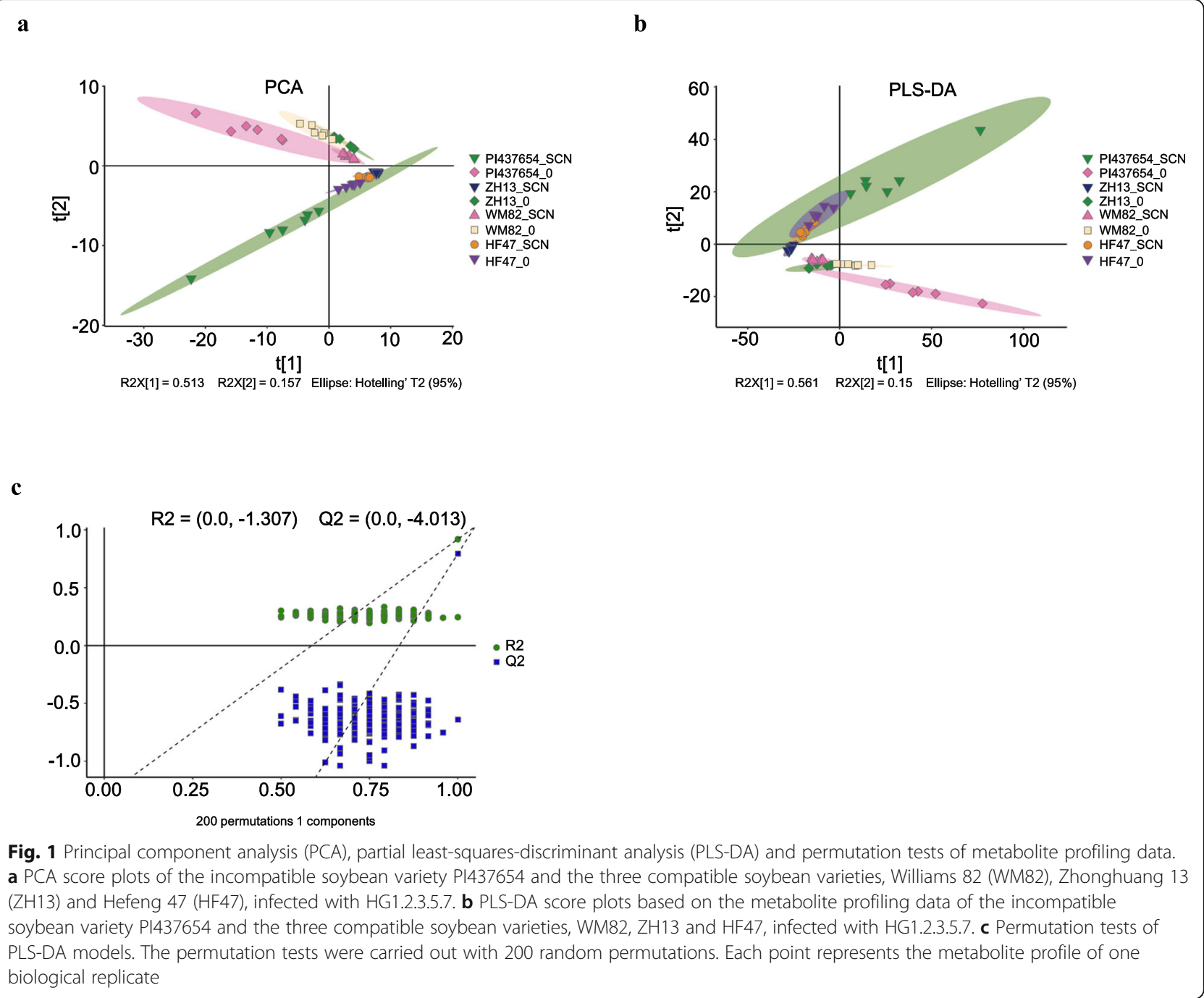

the incompatible soybean variety PI437654_SCN vs PI437654_0 and two compatible soybean varieties of ZH13_SCN vs ZH13_0 and HF47_SCN vs HF47_0, but was slightly up-regulated in the compatible soybean variety of WM82_SCN vs WM82_0. 16Hydroxyhexadecanoic acid was dramatically downregulated in the incompatible soybean variety PI437654_SCN vs PI437654_0 and two compatible soybean varieties of WM82_SCN vs WM82_0 and ZH13_SCN vs ZH13_0, but was obviously upregulated in the compatible soybean variety of HF47_SCN vs HF47_0 (Table 1, Fig. S3). In contrast, D,L-tryptophan was simultaneously downregulated in the three compatible soybean varieties but significantly up-regulated in the incompatible soybean variety PI437654_SCN vs PI437654_0 (Table 1). The results indicated that D,L-tryptophan had a role in defense metabolite production in the incompatible soybean variety PI437654 infected by HG1.2.3.5.7.

(2) Specific differential metabolites in the compatible soybean varieties. There were 18 significantly differential metabolites that were specifically present in the compatible soybean varieties and absent in the incompatible soybean variety PI437654_SCN vs PI437654_0 (Table 1, Fig. S3). Both 2-oxo-4-methylthiobutanoic acid and 4hydroxycoumarin were simultaneously overlapped and down-regulated in all the three compatible soybean varieties (Table 1, Fig. S3), while the other 16 metabolites were present in only one or two of the three compatible soybean varieties (Table 1, Fig. S3). Prunetin was specific to WM82_SCN vs WM82_0 and dramatically up-regulated, while trans-ferulic acid was specific to HF47_SCN vs HF47_0 and dramatically up-regulated (Table 1, Fig. 
Table 1 Differential metabolites in the roots of the incompatible soybean variety PI437654 and three compatible soybeans, WM82, ZH13 and HF47, infected with the HG1.2.3.5.7

\begin{tabular}{|c|c|c|c|c|}
\hline Metabolite & PI 437654 & WM82 & ZH13 & HF47 \\
\hline linolenic acid & $-1.604^{a}$ & -1.254 & -1.741 & -1.172 \\
\hline D-leucine & -0.759 & 0.322 & -2.313 & -1.289 \\
\hline 16-hydroxyhexadecanoic acid & -1.100 & -0.537 & -1.138 & 0.685 \\
\hline D,L-tryptophan & 1.300 & -0.544 & -0.892 & -0.556 \\
\hline D-aspartic acid & -8.796 & / & / & / \\
\hline linoleic acid & -6.199 & / & / & / \\
\hline N-palmitoyl alanine & -3.060 & / & / & / \\
\hline cycloleucine & -1.632 & / & / & / \\
\hline D,L-2,4-diaminobutyric acid & -1.307 & / & / & / \\
\hline phytosphingosine & -0.987 & / & / & / \\
\hline 10-oxo-nonadecanoic acid & -0.885 & / & / & / \\
\hline N-acetyltranexamic acid & 3.688 & / & / & / \\
\hline nicotine & 2.543 & / & / & / \\
\hline L-arginine & 0.893 & / & / & / \\
\hline pipecolinic acid & -2.026 & -1.591 & -1.390 & / \\
\hline gallocatechin gallate & -1.688 & -1.573 & / & / \\
\hline 4-methylquinoline & 1.110 & / & / & -0.584 \\
\hline nicotyrine & 1.096 & / & / & -0.563 \\
\hline L-trans-4-hydroxy-L-proline & 1.047 & / & / & -0.556 \\
\hline 2-oxo-4-methylthiobutanoic acid & / & -0.852 & -1.311 & -0.474 \\
\hline 4-hydroxycoumarin & / & -0.933 & -1.293 & -0.430 \\
\hline N-cyclohexanecarbonylpentadecylamine & / & -2.816 & -4.143 & / \\
\hline $\mathrm{PC}(\mathrm{O}-14: 0 / 2: 0)$ & / & -2.613 & / & 1.522 \\
\hline isoliquiritigenin & / & -2.000 & -3.391 & / \\
\hline rhamnazin & / & -1.037 & / & / \\
\hline betulinic acid & / & -1.020 & / & / \\
\hline drimenol & / & -0.923 & -2.296 & / \\
\hline 3-hydroxy-7-methoxyflavone & / & 2.170 & -7.687 & / \\
\hline prunetin & / & 1.637 & / & / \\
\hline baicalein & / & / & -1.560 & / \\
\hline ent-kaur-16-en-19-ol & / & / & / & -5.012 \\
\hline isopimaric acid & / & / & / & -3.091 \\
\hline communic acid & / & / & / & -2.529 \\
\hline hydroxy citronellal & / & / & / & -1.470 \\
\hline hexadecanamide & / & / & / & -1.324 \\
\hline dichotosinin & / & -2.044 & / & -1.212 \\
\hline trans-ferulic acid & / & / & / & 3.011 \\
\hline
\end{tabular}

Note: ${ }^{\text {a' }}$ represents the $\log _{2}$ FC value, which is the ratio of the average relative expression of metabolites in the incompatible soybean variety PI437654 and the three compatible soybean varieties, WM82, ZH13 and HF47, infected by HG1.2.3.5.7 compared with that in the controls innoculated with water. A positive value indicates that the differential metabolites are up-regulated, while a negative value indicates the differential metabolites are down-regulated. ' $/$ ' indicates that such metabolites were absent in the tested soybean varieties

S3). 3-Hydroxy-7-methoxyflavone was dramatically up-regulated in WM82_SCN vs WM82_0 but dramatically down-regulated in ZH13_SCN vs ZH13_0, while PC(O-14:0/2:0) was dramatically up- regulated in HF47_SCN vs HF47_0 but dramatically down-regulated in WM82_SCN vs WM82_0 (Table 1, Fig. S3). The other 14 metabolites were obviously down-regulated. N- 
cyclohexanecarbonylpentadecylamine, isoliquiritigenin and drimenol were simultaneously downregulated in WM82_SCN vs WM82_0 and ZH13_SCN vs ZH13_0, while dichotosinin was simultaneously down-regulated in WM82_SCN vs WM82_0 and HF47_SCN vs HF47_0 (Table 1, Fig. S3). Rhamnazin and betulinic acid were specific to WM82_SCN vs WM82_0, baicalein was specific to ZH13_SCN vs ZH13_0, while ent-kaur-16-en-19-ol, isopimaric acid, communic acid, hydroxy citronellal and hexadecanamide were specific to HF47_SCN vs HF47_0 (Table 1, Fig. S3). The down-regulated differential metabolites in the compatible soybean varieties were probably linked to basal defenses or physiological responses modulated by HG1.2.3.5.7 infection for the establishment and development of the feeding sites.

(3) Specific differential metabolites in the incompatible soybean variety PI437654. There were 10 differential metabolites specific to the incompatible soybean variety PI437654_SCN vs PI437654_0 (Table 1, Fig. S3), including seven down-regulated and three up-regulated metabolites (Table 1). DAspartic acid and linoleic acid were the top two significantly down-regulated metabolites, showing an approximately 70-fold reduction in PI437654_SCN vs PI437654_0 (Table 1), while N-palmitoyl alanine, cycloleucine and D,L-2,4-diaminobutyric acid were reduced approximately 8-, 3- and 2.5-fold, respectively, in PI437654_SCN vs PI437654_0 (Table 1). Among the three up-regulated metabolites, Nacetyltranexamic acid and nicotine were more abundant by approximately 12.9 - and 5.8 -fold in PI437654_SCN vs PI437654_0 (Table 1). In addition, five obviously differential metabolites, including two down-regulated (pipecolinic acid and gallocatechin gallate) and three up-regulated (4methylquinoline, nicotyrine and L-trans-4-hydroxyL-proline) metabolites were identified in PI437654_SCN vs PI437654_0. However, these five metabolites were not uniquely present in the incompatible soybean variety PI437654 and were also present in the compatible soybean varieties (Table 1). These results indicated that up-regulated $\mathrm{N}$ acetyltranexamic acid and nicotine might play potential roles in defense against HG1.2.3.5.7 infection in the incompatible soybean variety PI437654.

\section{Metabolic pathways of the differential metabolites in the incompatible and compatible soybean varieties}

The metabolic pathways of differential metabolites were analyzed by KEGG database searching. In total, 14 metabolic pathways were altered in the incompatible soybean variety PI437654_SCN vs PI437654_0, while nine, nine and eight metabolic pathways were altered in the compatible soybean varieties, WM82 SCN vs WM82_0, ZH13_SCN vs ZH13_0, HF47_ SCN vs HF47_0, respectively (Fig. 2, Table 2). The incompatible response-related KEGG pathways were the focuses of this study and were divided into the following two categories.

(1) Overlapping KEGG metabolic pathways between the incompatible and compatible soybean varieties. KEGG metabolic pathways including cutin, suberine and wax biosynthesis (ath00073), alpha-linolenic acid metabolism (ath00592) and the biosynthesis of unsaturated fatty acids (ath01040) were enriched and overlapped between the incompatible soybean variety PI437654 and the three compatible soybean varieties according to association with two differential metabolites, 16-hydroxyhexadecanoic acid and linolenic acid (Table 2). Moreover, the cutin, suberine and wax biosynthesis KEGG metabolic pathway (ath00073) was obviously activated in both the incompatible soybean variety PI437654 and the three compatible soybean varieties infected with HG1.2.3.5.7 (Fig. 2, Table 2), indicating its potential role in the basal defense response of various soybean varieties against HG1.2.3.5.7 infection.

(2) Specific KEGG metabolic pathways in the incompatible soybean variety PI437654. Nine specific KEGG metabolic pathways in the incompatible soybean variety PI437654 associated with three differential metabolites including Larginine, phytosphingosine and D-aspartic acid were identified (Table 2). The specific associated KEGG metabolic pathways included sphingolipid metabolism (ath00600), alanine, aspartate and glutamate metabolism (ath00250), arginine biosynthesis (ath00220), monobactam biosynthesis (ath00261), aminoacyl-tRNA biosynthesis (ath00970), arginine and proline metabolism (ath00330), biosynthesis of secondary metabolites-unclassified (ath00999), ABC transporters (ath02010) and biosynthesis of amino acids (ath01230) (Table 2). Although the tropane, piperidine and pyridine alkaloid biosynthesis KEGG metabolic pathway (ath00960) showed the most obvious difference in the incompatible soybean PI437654 (Fig. 2), it was not specific to this incompatible soybean variety, as it was also present in two compatible soybean varieties, WM82 and ZH13 (Table 2). Among these nine KEGG pathways, sphingolipid metabolism (ath00600), alanine, aspartate and glutamate metabolism (ath00250) and arginine biosynthesis (ath00220) were dramatically changed (Fig. 2; Table 2), suggesting their prevailing 


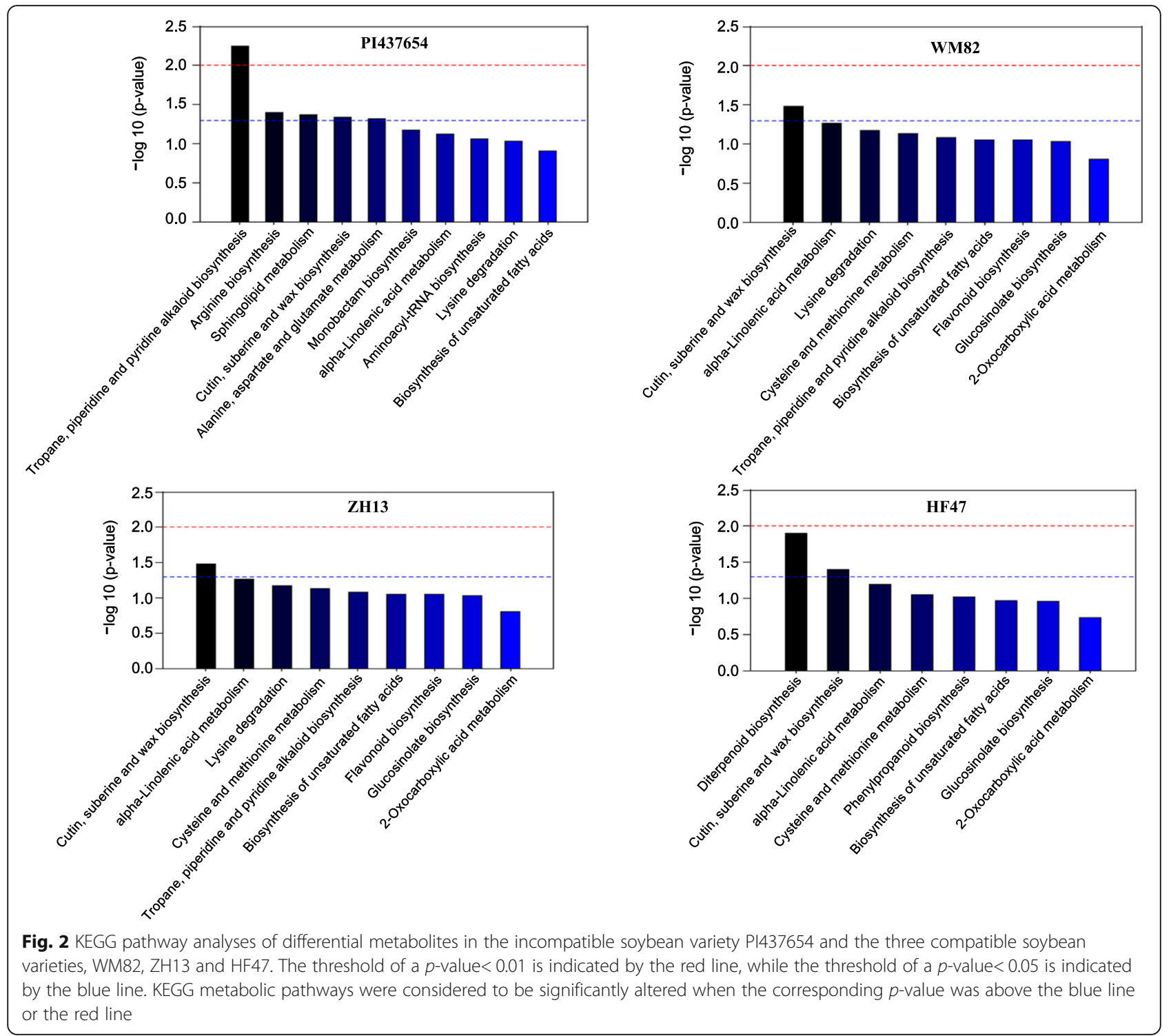

roles against HG1.2.3.5.7 infection in the incompatible soybean variety PI437654.

\section{Associated genes related to the up-regulated metabolites of the incompatible soybean variety PI437654}

To predict the genes associated with the up-regulated differential metabolites of the incompatible soybean variety PI437654, the transcriptome of the incompatible soybean variety PI437654 and the three compatible soybean varieties, WM82, ZH13 and HF47, as well as their corresponding controls, were sequenced, and these transcriptome data were combined with the metabolomic data. The comparative transcriptome analyses identified 15,835 DEGs (6922_UP vs 8913_DOWN), 12,225 DEGs (6001_UP vs 6224_DOWN), 18,362 DEGs (9589_UP vs 8773_DOWN) and 19,528 DEGs (8944_UP vs 10,584_ DOWN) in the incompatible soybean variety PI437654 and the three compatible soybean varieties, WM82, ZH13 and HF47, infected by HG1.2.3.5.7, respectively (Fig. S4). As mentioned above, three key metabolites (Nacetyltranexamic acid, nicotine and D,L-tryptophan) that were significantly up-regulated in the incompatible soybean variety PI437654 infected by HG1.2.3.5.7, were classified into two types. One type was specific to the incompatible soybean variety PI437654, and the other type was overlapped but simultaneously dramatically downregulated in the three compatible soybean varieties.

One of these types was the significantly up-regulated metabolites specifically present in the incompatible soybean variety PI437654, which included Nacetyltranexamic acid and nicotine. The results of combined analyses showed that 14 potentially associated genes (10 positive and four negative correlations) were simultaneously linked to $\mathrm{N}$-acetyltranexamic acid and 
Table 2 Analyses of the KEGG metabolic pathways associated with the differential metabolites in the incompatible soybean variety PI437654 and the three compatible soybean varieties, WM82, ZH13 and HF47, infected by HG1.2.3.5.7

\begin{tabular}{|c|c|c|c|c|c|c|}
\hline Metabolite & KEGG ID & Annotation & $\mathrm{PI} 437654$ & WM82 & ZH13 & HF47 \\
\hline 16-hydroxyhexadecanoic acid & ath00073 & cutin, suberine and wax biosynthesis & $1.337^{*}$ & 1.480 & 1.480 & 1.402 \\
\hline \multirow[t]{2}{*}{ linolenic acid $^{a}$} & ath00592 & alpha-linolenic acid metabolism & 1.130 & 1.272 & 1.272 & 1.195 \\
\hline & ath01040 & biosynthesis of unsaturated fatty acids & 0.914 & 1.052 & 1.052 & 0.977 \\
\hline \multirow[t]{3}{*}{ 2-oxo-4-methylthiobutanoic acid ${ }^{a}$} & ath00270 & cysteine and methionine metabolism & / & 1.134 & 1.133 & 1.058 \\
\hline & ath00966 & glucosinolate biosynthesis & / & 1.036 & 1.036 & 0.961 \\
\hline & ath01210 & 2-oxocarboxylic acid metabolism & / & 0.808 & 0.808 & 0.735 \\
\hline isoliquiritigenin & ath00941 & flavonoid biosynthesis & / & 1.052 & 1.052 & / \\
\hline isopimaric acid & ath00904 & diterpenoid biosynthesis & / & / & / & 1.906 \\
\hline \multicolumn{7}{|l|}{ ent-kaur-16-en-19-ol } \\
\hline trans-ferulic acid & ath00940 & phenylpropanoid biosynthesis & / & / & / & 1.025 \\
\hline phytosphingosine & ath00600 & sphingolipid metabolism & 1.370 & / & / & / \\
\hline D-aspartic acid & ath00250 & alanine, aspartate and glutamate metabolism & 1.321 & / & / & / \\
\hline \multirow[t]{7}{*}{ L-arginine $^{a}$} & ath00220 & arginine biosynthesis & 1.405 & / & / & / \\
\hline & ath00261 & monobactam biosynthesis & 1.181 & / & / & / \\
\hline & ath00970 & aminoacyl-tRNA biosynthesis & 1.060 & / & / & / \\
\hline & ath00330 & arginine and proline metabolism & 0.893 & / & / & / \\
\hline & ath00999 & biosynthesis of secondary metabolites - unclassified & 0.792 & / & / & / \\
\hline & ath02010 & $\mathrm{ABC}$ transporters & 0.700 & / & / & / \\
\hline & ath01230 & biosynthesis of amino acids & 0.693 & / & / & / \\
\hline nicotine & ath00960 b & tropane, piperidine and pyridine alkaloid biosynthesis & 2.253 & / & / & / \\
\hline pipecolinic acid $^{a}$ & & & & 1.088 & 1.088 & / \\
\hline pipecolinic acid ${ }^{a}$ & ath00310 & lysine degradation & 1.037 & 1.177 & 1.177 & / \\
\hline
\end{tabular}

Note: ${ }^{\prime * \prime}$ represents the $\log _{10}$ ( $p$-value) value, while ' $p$-value' represents the $p$-value of each KEGG metabolic pathway. The smaller the $p$-value is, the greater the - $\log \left(p\right.$-value). ${ }^{\prime a}$ indicates that the same differential metabolite is involved in at least two different KEGG metabolic pathways. "b' indicates that two different differential metabolites are involved in the same KEGG metabolic pathway

nicotin, while 68 (52 positive and 16 negative correlations) and 54 (21 positive and 33 negative correlations) associated genes were specifically linked to either nicotin or $\mathrm{N}$-acetyltranexamic acid, respectively (Fig. 3a, Table S3). These associated genes were subjected to GO analyses. The most abundant GO terms in the biological processes category were "translation", followed by "cytoplasmic translation" and "rRNA modification" (Fig. S5, Table S4). Regarding the cellular component category, the most abundant GO terms were "ribosome", "cytosolic ribosome", "cytosolic small ribosomal subunit", cytosolic large ribosomal subunit" and "nucleolus" (Fig. S5, Table S4). Concerning the molecular function category, the most abundant GO terms were "structural constituent of ribosome", "mRNA binding", "rRNA binding", "RNA binding" and "inorganic cation transmembrane transporter activity" (Fig. S5, Table S4). Accordingly, the associated genes were enriched in the KEGG pathways involving the largest numbers of unigenes, which were "ribosome", followed by "RNA degradation", "ribosome biogenesis in eukaryotes", "peroxisome" and "autophagy" (Fig. 4a, Table S5).
The other type was the significantly up-regulated metabolite in the incompatible soybean variety PI437654 that were simultaneously dramatically down-regulated in the three compatible soybean varieties, which included D,L-tryptophan. Among the top 150 most associated genes, 80 genes exhibited positive correlations, while 70 genes exhibited negative correlations (Fig. 3b, Table S6). Among these associated genes, the most abundant GO terms in the biological processes category were "response to chitin", followed by the "ethylene-activated signaling pathway" and "defense response" (Fig. S5, Table S7). Regarding the cellular component category, the most abundant GO terms were "extracellular region", "apoplast" and "photosystem I" (Fig. S5, Table S7). Concerning the molecular function category, the most abundant GO terms were "transcription factor activity, sequencespecific DNA binding", "heme binding" and "hydroquinone:oxygen oxidoreductase activity" (Fig. S5, Table S7). Accordingly, the associated genes were enriched in the KEGG pathways involving the largest numbers of unigenes, which were "plant hormone 


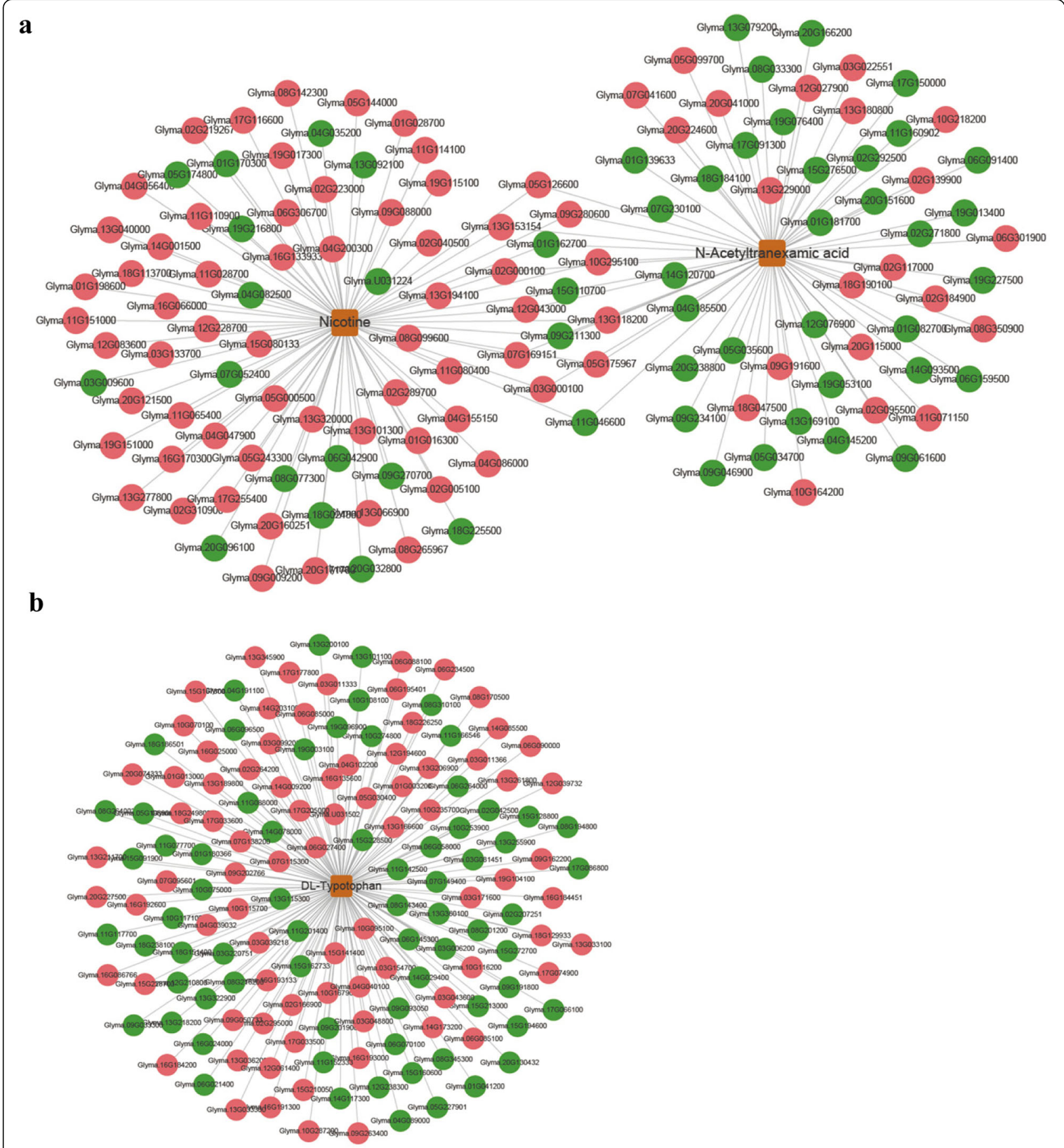

Fig. 3 Associated genes related to the significantly up-regulated metabolites in the incompatible soybean variety PI437654 and their associated networks. a Genes associated with the significantly up-regulated metabolites specifically present in the incompatible soybean variety PI437654. $\mathbf{b}$ Genes associated with the significantly up-regulated metabolite D,L-tryptophan, which was significantly up-regulated in the incompatible soybean variety PI437654 but simultaneously dramatically down-regulated in the three compatible soybean varieties, WM82, ZH13 and HF47. Orange squares represent the significantly up-regulated metabolites. Red circles represent positive correlations, while green circles represent negative correlations 


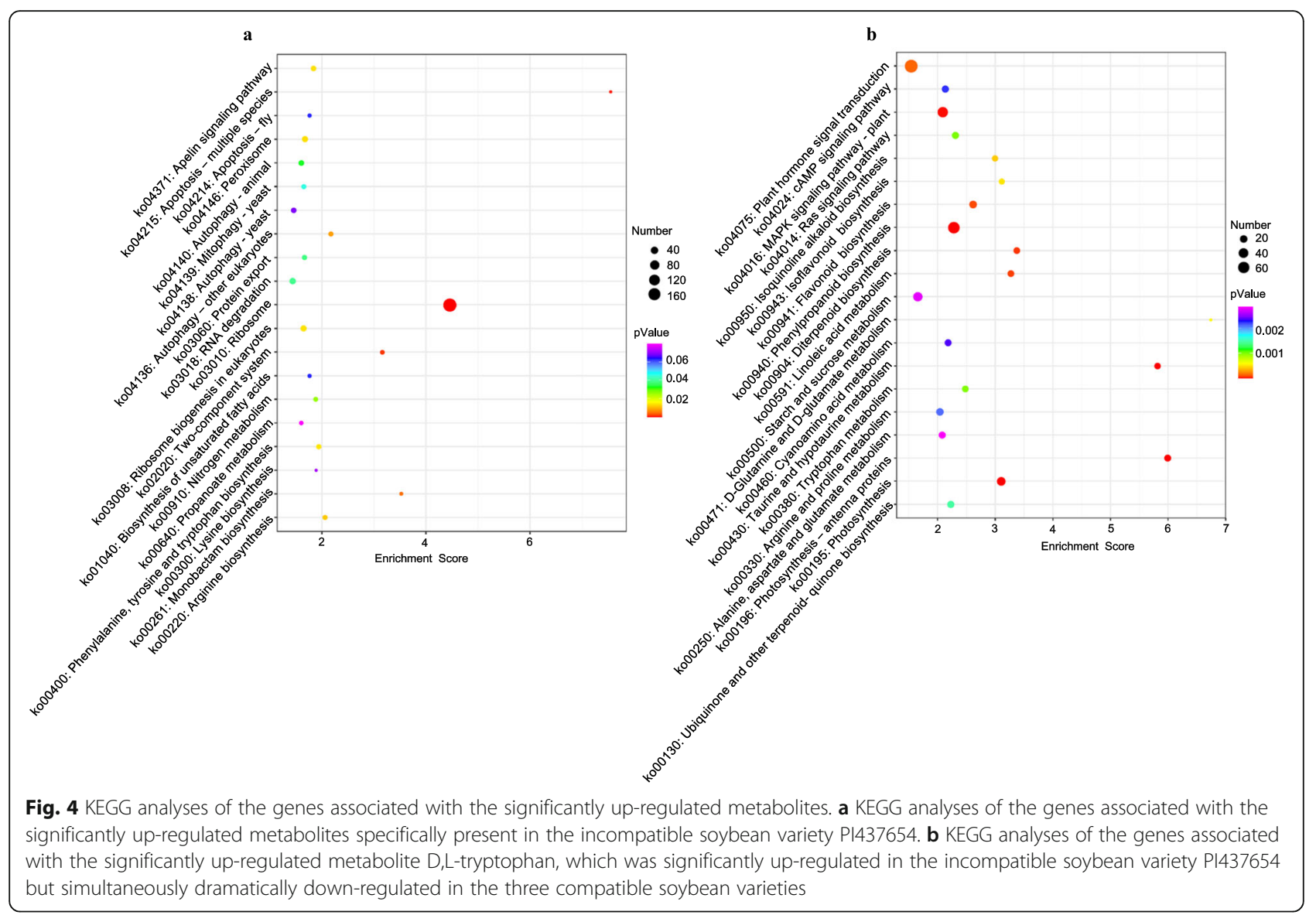

signal transduction", followed by "phenylpropanoid biosynthesis", "MAPK signaling pathway-plant", "starch and sucrose metabolism" and "photosynthesis" (Fig. 4b, Table S8).

These results of the combined metabolomic and transcriptomic analyses revealed potential genes associated with the most significantly up-regulated metabolites of the incompatible soybean variety PI437654 and suggested the likely corresponding biological processes, cellular components, molecular functions and KEGG pathways.

\section{Discussion}

In this study, the incompatible soybean variety PI437654 and the three compatible soybean varieties, WM82, ZH13 and HF47, were used to explore the changes in metabolites in soybean roots in response to HG1.2.3.5.7 via LC/MS analyses. In total, 37 differential metabolites and 20 KEGG metabolic pathways were identified, which were divided into three categories: metabolites/metabolic pathways common among all the tested incompatible and compatible soybean varieties; metabolites/metabolic pathways specific to the compatible soybean varieties; metabolites/metabolic pathways specific to the incompatible soybean varieties (Tables 1 and 2). It was found that certain differential metabolites, such as linolenic acid and L-arginine, were probably involved in more than one KEGG metabolic pathway, while other differential metabolites, such as nicotine and pipecolinic acid, were likely involved in the overlapping KEGG metabolic pathways (tropane, piperidine and pyridine alkaloid biosynthesis pathways) (Table 2). However, only 12 differential metabolites (32\%) were found to be involved in the predicted KEGG metabolic pathways.

In this study, some basal defense-related potential metabolites, such as linolenic acid and 16hydroxyhexadecanoic acid, were found in the incompatible soybean variety PI437654 and the three compatible soybean varieties (WM82, ZH13 and HF47) infected by HG1.2.3.5.7, and our metabolomic analysis results were consistent with some previous reports [39-41]. Linolenic acid is involved in alpha-linolenic acid metabolism and the biosynthesis of unsaturated fatty acids [39-43]. Linolenic acid has been found to be associated with abiotic stress responses, such as cold tolerance in wheat [3941]. Unsaturated fatty acids are considered as a new generation of plant resistance inducers [42, 43]. Amruthesh et al. found that the application of unsaturated fatty acids induced strong resistance to downy mildew in pearl millet [42]. Clay et al. found that the higher 
contents of unsaturated fatty acids in the nuclear membrane of the resistant species in Gossypium barbadense was related to host resistance [43].

The metabolites and associated KEGG metabolic pathways specific to the incompatible soybean variety PI437654 infected with HG1.2.3.5.7 probably play pivotal roles in plant stress, tolerance or resistance based on the related literature [44-61]. L-arginine is the precursor of nitric oxide (NO), and $\mathrm{NO}$ is synthesized from Larginine by NO synthase in the L-arginine-NO pathway. $\mathrm{NO}$ is widely considered as an important regulator of cell function and communication in physiological and pathophysiological states [44-46]. NO is a second messenger in the stress response and is involved in plant responses to biotic and abiotic stresses [47, 48]. Larginine is linked to and involved in many KEGG metabolic pathways, such as arginine and proline metabolism, aminoacyl-tRNA biosynthesis and $\mathrm{ABC}$ transporter pathways. Previous studies have indicated that there is a positive correlation between proline accumulation and plant tolerance [49]. Aminoacyl-tRNA synthetases catalyze the connection between amino acids and their homologous tRNAs and constitute a specific set of enzymes ensuring the fidelity of the transfer of genetic information from DNA to proteins [50, 51]. ABC transporters transport material cross membranes by using the energy from ATP hydrolysis [52]. Recently, ABC transporters have attracted increasing attention due to their role in pesticide resistance [53]. Phytosphingosine (PHS) is a member of the sphingosine family that shows fungicidal activity [54]. Castro et al. found that PHS reduced the viability of cells and induced apoptosis in Neurospora crassa [55]. D-Aspartic acid participates in alanine, aspartate and glutamate metabolism, while glutamate dehydrogenase $(\mathrm{GDH})$ is a central enzyme in glutamate metabolism [56]. The enhancement of drought resistance in wheat is related to ammonia assimilation and increased NADH-GDH activity under low or high osmotic stress [61]. Nicotine is widely used to kill many kinds of harmful insects [58]. Vänninen at el discovered that nicotine had a strong killing effect on the adults of Miridae species [60]. Nicotine is involved in the biosynthesis of tropane, piperidine and pyridine alkaloids via metabolic pathways [62] and alkaloids have been found to exert obvious inhibitory effects on the sunflower downy mildew fungus Plasmopara halstedii [59]. Thus, Larginine, phytosphingosine, D-aspartic acid and nicotine may be involved in the incompatible response of soybean against soybean cyst nematodes.

In contrast, the differential metabolite 2-oxo-4methylthiobutanoic acid, which showed overlap among all three compatible soybean varieties but was absent in incompatible soybean variety, was predicted to participate in three KEGG metabolic pathways, including cysteine and methionine metabolism, glucosinolate biosynthesis and 2-oxocarboxylic acid metabolism. Moreover, 2-oxo-4-methylthiobutanoic acid was simultaneously down-regulated in the three compatible soybean varieties (ZH13, WM82 and HF47) infected with HG1.2.3.5.7, which likely resulted in decreased biosynthesis of glucosinolate. It has been reported that glucosinolate is a plant secondary metabolite that exerts a preventive effect against plant pathogens and soil-borne plant pests $[63,64]$. Additionally, the KEGG metabolic pathways of flavonoid biosynthesis and diterpenoid biosynthesis, which are reported to be involved in plant resistance to pathogens, were found to be enriched in one or two compatible soybean varieties $[65,66]$. These types of differential metabolites and their related KEGG metabolic pathways might play roles in the manipulation of host innate immunity to establish and maintain feeding sites for the development of soybean cyst nematodes $[67,68]$. In this study, a local metabolite-calibrated database was used to identify potential differential metabolites, which ensured the accuracy of the results [16, 69, 70]. However, this database also presents potential disadvantages because of lacking many important compounds due to its limited capacity. In this study, the different metabolomic responses of incompatible and compatible soybean varieties to soybean cyst nematode infection were thoroughly characterized, and key differential metabolites and related KEGG metabolic pathways were identified. The likely functions of differential metabolites and related KEGG metabolic pathways were predicted.

In this study, three key metabolites ( $\mathrm{N}$-acetyltranexamic acid, nicotine and D,L-tryptophan) that were significantly up-regulated in the incompatible soybean variety PI437654 infected by HG1.2.3.5.7, were identified and classified into two types. The combination of metabolomic analyses with transcriptomic expression profiling aided in predicting potential genes associated with the most significantly up-regulated metabolites of the incompatible soybean variety PI437654 and suggested the likely corresponding biological processes, cellular components, molecular functions and pathways.

\section{Conclusions}

In this study, the main metabolomic differences between the incompatible soybean variety PI437654 and three compatible soybean varieties infected with HG1.2.3.5.7 were characterized. In total, 37 differential metabolites and 20 KEGG metabolic pathways were identified, which were divided into three categories: metabolites/metabolic pathways overlapped among all the tested incompatible and compatible soybean varieties; metabolites/metabolic pathways specific to compatible soybean varieties; metabolites/metabolic pathways specific to incompatible soybean variety. Twelve differential metabolites were 
found to be involved in the predicted KEGG metabolic pathways. Moreover, 14 differential metabolites such as significantly up-regulated nicotine and down-regulated D-aspartic acid, and their associated KEGG pathways such as tropane, piperidine and pyridine alkaloid biosynthesis, alanine, aspartate and glutamate metabolism, sphingolipid metabolism and arginine biosynthesis were significantly altered and abundantly enriched specifically in the incompatible soybean variety PI437654 and likely played pivotal roles in defending against HG1.2.3.5.7. Moreover, the combination of metabolomic analyses with transcriptomic expression profiling aided in predicting potential associated genes related to the most significantly up-regulated metabolites of the incompatible soybean variety PI437654, and suggested the likely biological processes, cellular components, molecular functions and pathways. To the best of our knowledge, our study was the first to investigate the different responses of incompatible and compatible soybean varieties infected with soybean cyst nematodes by combining metabolomic analyses and transcriptomics. Our results not only identified potential novel metabolites and associated genes involved in the incompatible response of PI437654 to the soybean cyst nematode HG1.2.3.5.7, but also provided new insights into the interactions between soybeans and soybean cyst nematodes.

\section{Methods}

Soybean seedling preparation and inoculation of soybean cyst nematodes

The seeds of the incompatible soybean variety PI437654 and the three compatible soybean varieties, WM82, ZH13 and HF47, were obtained from China National Gene Bank (http://www.nationalgenebank.org/). Soybean seeds were sterilized by soaking in a $1.0 \%(\mathrm{w} / \mathrm{v}) \mathrm{NaClO}$ solution for $5 \mathrm{~min}$ [71], and the seeds were then washed with sterilized water to remove residual $\mathrm{NaClO}$. After surface sterilization, the seeds were placed between moist sterilized filter papers at $26^{\circ} \mathrm{C}$. After approximately $48 \mathrm{~h}$, the germinated seeds were transplanted into a soil mixture consisting of $75 \%(\mathrm{v} / \mathrm{v})$ soil and $25 \%(\mathrm{v} / \mathrm{v})$ sand and cultured in a greenhouse, where the day/night temperatures were set to $28{ }^{\circ} \mathrm{C} / 26^{\circ} \mathrm{C}$ with a photoperiod

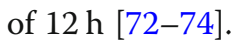

Single cysts of HG1.2.3.5.7 were cultured on the roots of the compatible soybean variety $\mathrm{ZH} 13$ grown in an autoclaved soil mixture in a greenhouse at $28^{\circ} \mathrm{C} / 26^{\circ} \mathrm{C}$ with a photoperiod of $12 \mathrm{~h}$ [13, 73]. The cysts were harvested and placed on 500 -mesh $(25 \mu \mathrm{m})$ sieves in $3 \mathrm{mM} \mathrm{ZnCl} 2$ solution and then incubated in the dark at $25^{\circ} \mathrm{C}[38,73,75]$. The hatched J2s were collected with a glass tube [76]. Fourteen-day-old soybean seedlings were inoculated with $1000 \mathrm{~J} 2 \mathrm{~s} /$ seedling. At 8 days post inoculation (dpi), the root samples of the incompatible soybean variety PI437654 and the three compatible soybean varieties, WM82, ZH13 and HF47, as well as their corresponding controls were harvested. Each type of root sample was divided into two parts for the following metabolomic and transcriptomic analyses.

\section{Juveniles staining and cysts counting}

Root samples of the incompatible soybean variety PI437654 and the three compatible soybean varieties, WM82, ZH13 and HF47, were collected at $8 \mathrm{dpi}$, rinsed in water, and then soaked in a $5.25 \% \mathrm{NaClO}$ solution for $5 \mathrm{~min}$. The soybean samples were then rinsed twice in water for $10 \mathrm{~min}$ and $15 \mathrm{~min}$, respectively. The soybean samples were dropped into a boiling $0.01 \%$ acid fuchsin solution for $30 \mathrm{~s}$ and then rinsed in water after cooling. The samples were transferred to and stored in glycerol [77-80]. The morphology of the HG1.2.3.5.7 nematodes was observed, and their numbers within different soybean roots were counted with a microscope (OLYMPUS-SZ2-ILST).

At $60 \mathrm{dpi}$, samples of the soil mixture in which the soybean plants were grown were suspended in water, and the soybean roots were processed by washing and rubbing to dislodge the females and cysts. The cysts were extracted by sieving the suspension through nested 20 -mesh $(850 \mu \mathrm{m}$, on the top) over 60 -mesh $(250 \mu \mathrm{m}$, on the bottom) sieves [81, 82]. The numbers of cysts, which were recorded in the form of cysts per plant $[81,82]$, were counted with a stereoscopic microscope (OLYMPUS-BX53F).

\section{Untargeted metabolomics using LC/MS}

At $8 \mathrm{dpi}$, the root samples of the incompatible soybean variety PI437654 and the three compatible soybean varieties, WM82, ZH13 and HF47, as well as their corresponding controls were harvested and named PI437654_SCN, PI437654_0, WM82_SCN, WM82_0, ZH13_SCN, ZH13_0, HF47_SCN and HF47_0, respectively. Each soybean root sample was ground into a powder in liquid nitrogen. An $80 \mathrm{mg}$ sample of root powder was then dropped into a 1.5 $\mathrm{mL}$ centrifuge tube. Then, $20 \mu \mathrm{L}$ of an internal standard $(0.3 \mathrm{mg} / \mathrm{mL} \mathrm{L}-2$ - chlorophenylalanine) and $1 \mathrm{~mL}$ of $70 \%$ methanol (methanol:water $=7: 3$ (volume ratio)) were added to the tube. The tube was placed at $20^{\circ} \mathrm{C}$ for precooling, and the mixture was ground with a grinding machine for $2 \mathrm{~min}$ at $60 \mathrm{~Hz}$. Then, ultrasonic extraction was performed at $4{ }^{\circ} \mathrm{C}$ for 30 min. After standing at $-20^{\circ} \mathrm{C}$ for $20 \mathrm{~min}$, the sample was centrifuged for $15 \mathrm{~min}$ at $13,000 \mathrm{rpm}$ at $4{ }^{\circ} \mathrm{C}$, and 
$200 \mu \mathrm{L}$ of the supernatant was harvested with a syringe. This procedure was repeated for each sample three times, and the supernatants from the same source were combined. After filtering through a $0.22 \mu \mathrm{m}$ organic-phase pinhole filter, the corresponding supernatant was subjected to LC-MS analysis. LCMS analysis was performed on an Agilent 1290 Series UHPLC system coupled to an Agilent 6540 TOF/MS instrument with a Dual AJS ESI source. Each treatment had six repetitions $[16,83]$.

\section{Metabolomic analyses}

The metabolites were identified by using a local metabolite-calibrated database that contained about 500 metabolites based on the standards of peak information involving retention time and high-precision mass values $[16,69,70]$. Multivariate statistical analyses, including principal component analysis (PCA) and partial least squares discriminant analysis (PLSDA), were performed using SIMCA- $\mathrm{P}^{+} 14.0$ version software [84] to distinguish the differences in metabolic profiles between the incompatible soybean variety PI437654 and the three compatible soybean varieties, WM82, ZH13 and HF47, infected by HG1.2.3.5.7. The Hotelling's T2 region, shown as an ellipse in the score plots of the models, defined the $95 \%$ confidence interval of the modeled variation. According to the T-test, a variable with a $p$-value $<0.05$ was considered as a significantly different variable [83]. Metabolites with VIP (variable importance in the projection) values $>1.0$ and $p$-values $<0.05$ were selected as differential metabolites [83]. KEGG metabolic pathways were predicted from the differential metabolites showing a $p$-value $<0.05$ [83].

\section{Transcriptomic analyses}

Root samples of the same six plants were sampled and randomly divided into two groups for each soybean variety, representing two biological repeats. The total RNA of each sample was extracted using a Plant RNA Kit (TIANGEN, China). Total RNA was quantified and analyzed for quality with an Agilent 2100 Bioanalyzer (Agilent, USA) [85]. One microgram of total RNA with a RNA integrity number (RIN) value above 8.0 was used for the subsequent preparation of libraries [86].

To construct a library $[78,85,86]$, mRNA was enriched and purified with oligo (dT)-rich magnetic beads and then broken into short fragments. Firstand second-strand cDNAs were synthesized by using the cleaved mRNA fragments as templates. An AxyPrep Mag PCR Clean-up kit (Axygen, USA) was used to further purify the double-stranded cDNA, and End
Prep Enzyme Mix (NEB, USA) was used to add adaptors. An AxyPrep Mag PCR Clean-up kit (Axygen, USA) was used to recover fragments of $\sim 450 \mathrm{bp}$. The PCR products were purified, and then the purified PCR products were validated with an Agilent 2100 Bioanalyzer (Agilent, USA) and quantified via Qubit and real-time PCR analysis (Applied Biosystems, USA). RNA sequencing was carried out using a $2 \times$ 150 paired-end $(\mathrm{PE})$ configuration with an Illumina Novaseq 6000 system.

Clean data were obtained by removing the reads that contained adapters and ploy- $\mathrm{N}$ sequences and low-quality reads from the raw data $[85,86]$. Reference genome and gene model annotation files were downloaded from the SoyBase website (https:// soybase.org/GlycineBlastPages/blast_descriptions.php). The paired-end clean reads were aligned to the reference genome by using HISAT [35]. HTSeq was used to calculate the numbers of reads mapped to each gene. The expression of genes was measured in fragments per $\mathrm{kb}$ per million fragments (FPKM) values. Genes showing an absolute $\log _{2}$ FC (fold change) $>0.5$ and a pvalue $<0.05$ were defined as DEGs, and a software DESeq2 was used to calculate significance [87]. GO (Gene Ontology) analyses were performed by using Blast2GO [86, 88]. KOBAS 2.0 was used to test the statistical enrichment of DEGs in KEGG pathways [35].

\section{Correlation analyses between metabolome and transcriptome data}

Correlation analyses were carried out as previously described [85, 89]. Briefly, Pearson correlation coefficients were calculated for the integration of the metabolome and transcriptome data. The mean contents of metabolites in the incompatible soybean variety PI437654 and the three compatible soybean varieties, WM82, ZH13 and HF47, and the mean transcript abundance of the DEGs together were calculated. One-way analysis of variance (ANOVA) associated with T-test was performed to determine significant differences between groups according to a $p$-value $<0.05$. PCA of the total transcriptomic dataset was performed in $\mathrm{R}$ ( $\mathrm{R}$ Core Team) [35]. The coefficients were calculated from the $\log _{2} \mathrm{FC}$ value of each metabolite and the $\log _{2} \mathrm{FC}$ value of each transcript. Correlations were determined according to an $\mathrm{R}^{2}$ coefficient $>0.9$. The relationships between the metabolome and transcriptome data were illustrated by using Cytoscape. The top 150 associated genes were the focuses of subsequent analyses based on the numbers of genes and the complexity of the network involved. 


\section{Abbreviations}

ABC transporters: ATP binding cassette transporters; ANOVA: Analysis of variance; DEGs: Differential expressed genes; dpi: Days post inoculation; FC: Fold change; FPKM: Fragments per kilobase million; GDH: Glutamate dehydrogenase; GO: Gene Ontology; GWAS: Genome-wide association studies; HF47: Hefeng 47; J2s: Juveniles of second stage; KEGG: Kyoto encyclopedia of genes and genomes; LC/MS: Liquid chromatographytandem mass spectrometry; MAPK: Mitogen-activated protein kinase; NADHGDH: Reduced form of nicotinamide-adenine dinucleotid-glutamate dehydrogenase; NO: Nitric oxide; PC: Polycarbonate; PCA: Principal component analysis; PCR: Polymerase chain reaction; PE: Paired-end;

PHS: Phytosphingosine; PLS-DA: Partial least squares-discrimination analysis; RIN: RNA integrity number; SCN: Soybean cyst nematode; TOF/MS: Time-offlight mass spectrometer; UHPLC: Ultra high performance liquid chromatography; VIP: Variable important in projection; vs: Versus; WM82: Williams 82; ZH13: Zhonghuang 13

\section{Supplementary Information}

The online version contains supplementary material available at https://doi. org/10.1186/s12870-021-02998-4

Additional file 1: Fig. S1. Development of HG1.2.3.5.7 juveniles in the roots of the incompatible soybean variety PI437654 and the three compatible soybean varieties, WM82, ZH13 and HF47. The root samples of the incompatible soybean variety PI437654 and the three compatible soybean varieties, WM82, ZH13 and HF47, infected by HG1.2.3.5.7 were collected at $8 \mathrm{dpi}$ and then stained with $0.0 .1 \%$ acid fuchsin solution. The juveniles of HG1 2.3.5.7 within soybean roots were examined by a microscopy. The bar represents $200 \mu \mathrm{m}$.

Additional file 2: Fig. S2. PCA and PLS-DA of the incompatible soybean variety PI437654 and the three compatible soybean varieties, WM82, ZH13 and HF47, infected with HG1.2.3.5.7 at 8 dpi compared with the results for controls innoculated with water. This chart was generated from the same data represented in Fig. 1, but they were displayed in a different form to show clear separation trends.

Additional file 3: Fig. S3. Venn diagrams showing the commonality and uniqueness of the differential metabolites between the incompatible soybean variety PI437654 and the three compatible soybean varieties, WM82, ZH13 and HF47, infected with HG1.2.3.5.7 at 8 dpi compared with the results for controls innoculated with water. The values represent the amounts of specific or overlapping different metabolites.

Additional file 4: Fig. S4. Transcriptomic profiles of the number of differentially expressed genes (DEGs) between the incompatible soybean variety PI437654 and the three compatible soybean varieties, WM82, ZH13 and HF47, infected with HG1.2.3.5.7 at 8 dpi compared with the results for controls innoculated with water, respectively. Red represents the number of up-regulated DEGs, while blue represents the number of the down-regulated DEGs.

Additional file 5: Fig. S5. GO analyses of the genes associated with the significantly up-regulated metabolites. (a) $\mathrm{GO}$ analyses of the genes associated with the significantly up-regulated metabolites specifically present in the incompatible soybean variety PI437654. (b) GO analyses of the genes associated with the significantly up-regulated metabolite D,L-tryptophan, which was significantly up-regulated in the incompatible soybean variety PI437654 but simultaneously dramatically down-regulated in the three compatible soybean varieties.

Additional file 6: Table S1. Numbers of SCN juveniles and cysts in the incompatible soybean variety PI437654 and the three compatible soybean varieties, WM82, ZH13 and HF47, infected by HG1.2.3.5.7.

Additional file 7: Table S2. Cumulative interpretation rate of the model in the incompatible soybean variety PI437654 and the three compatible soybean varieties, WM82, ZH13 and HF47, infected by HG1.2.3.5.7.

Additional file 8: Table S3. List of the genes associated with the significantly up-regulated metabolites specifically present in the incompatible soybean variety PI437654.
Additional file 9: Table S4. GO analyses of the genes associated with the significantly up-regulated metabolites specifically present in the incompatible soybean variety PI437654.

Additional file 10: Table S5. KEGG analyses of the genes associated with the significantly up-regulated metabolites specifically present in the incompatible soybean variety PI437654.

Additional file 11: Table S6. List of the genes associated with the significantly up-regulated metabolite D,L-tryptophan, which was significantly up-regulated in the incompatible soybean variety PI437654 but simultaneously dramatically down-regulated in the three compatible soybean varieties.

Additional file 12: Table S7. GO analyses of the genes associated with the metabolite D,L-tryptophan, which was significantly up-regulated in the incompatible soybean variety PI437654 but simultaneously dramatically down-regulated in the three compatible soybean varieties.

Additional file 13: Table S8. KEGG analyses of the genes associated with the metabolite D,L-tryptophan, which was significantly up-regulated in the incompatible soybean variety PI437654 but simultaneously dramatically down-regulated in the three compatible soybean varieties.

\section{Acknowledgements}

We thank to Dr. Peng Huan from Institute of Plant Protection, Chinese Academy of Agricultural Sciences, for the suggestions on the methods and revision. We thank to Dr. Yuqi Hao from Shanghai OE Biotech Co., Ltd., Shanghai, China for data analysis support. We thank to China National Gene Bank for soybean varieties support. We are grateful to Dr. Vijai Bhadauria from China Agricultural University for critical proofreading.

\section{Authors' contributions}

LAK and DLP conceived the study; XS and LAK wrote the manuscript. XS, QSC and JJW performed the experiments; XS and QSC conducted statistical analyses; SML revised the manuscript. All of the authors discussed the results, and commented on the manuscript, and approved its submission for potential publication.

\section{Funding}

This project was supported by the Natural Science Foundation of China (31872924), the Key Scientific and Technological Project of Henan Province (192102110003), and the National Technology System of Soybean Industry (CARS-04-PS05). The funders had no role in the study design, data collection and analyses, data interpretation, or in the writing of the manuscript.

\section{Availability of data and materials}

The soybean varieties used in this study were obtained from China National Gene Bank (http://www.nationalgenebank.org/). The data that support the findings of this study have been deposited in the CNSA (https://db.cngb.org/ cnsa/) of CNGBdb (China National GeneBank) with accession number CNP0001271.

\section{Declarations}

Ethics approval and consent to participate

Not applicable.

\section{Consent for publication}

Not applicable.

\section{Competing interests}

The authors declare that they have no competing interests.

\section{Author details}

${ }^{1}$ State Key Laboratory for Biology of Plant Diseases and Insect Pests, Institute of Plant Protection, Chinese Academy of Agricultural Sciences, Beijing 100193, China. ${ }^{2}$ Zhengzhou Tobacco Research Institute of CNTC, Zhengzhou, Henan, China. ${ }^{3}$ Soybean Research Institute, Heilongjiang Academy of Agricultural Sciences, Harbin 150086, China. 


\section{Received: 7 September 2020 Accepted: 30 April 2021}

\section{Published online: 14 May 2021}

\section{References}

1. Cook DE, Lee TG, Guo X, Melito S, Wang K, Bayless AM, et al. Copy number variation of multiple genes at Rhg1 mediates nematode resistance in soybean. Science. 2012;338(6111):1206-9. https://doi.org/10.1126/ science.1228746.

2. Liu S, Kandoth PK, Warren SD, Yeckel G, Heinz R, Alde J, et al. A soybean cyst nematode resistance gene points to a new mechanism of plant resistance to pathogens. Nature. 2012;492(7428):256-60. https://doi.org/10.1 038/nature11651.

3. Niblack TL, Lambert KN, Tylka GL. A model plant pathogen from the kingdom animalia: Heterodera glycines, the soybean cyst nematode. Annu Rev Phytopathol. 2006;44(1):283-303. https://doi.org/10.1146/annurev. phyto.43.040204.140218.

4. Masonbrink R, Maier TR, Muppirala U, Seetharam AS, Lord E, Juvale PS, et al. The genome of the soybean cyst nematode (Heterodera glycines) reveals complex patterns of duplications involved in the evolution of parasitism genes. BMC Genomics. 2019;20(1):119. https://doi.org/10.1186/s12864-0195485-8.

5. Duan Y, Zheng Y, Chen L, Zhou X, Wang Y, Sun J. Effects of abiotic environmental factors on soybean cyst nematode. Agric Sci China. 2009;8(3): 63-71.

6. Jones MGK. The development and function of plant cells modified by endoparasitic nematodes. Plant Parasitic Nematodes (Volume III). New York: Academic Press Inc. 1981;255-79.

7. Matthews KBF, Klink VP. Emerging approaches to broaden resistance of soybean to soybean cyst nematode as supported by gene expression studies. Plant Physiol. 2009;151(3):1017-22.

8. Hu Y, You J, Li C, Williamson VM, Wang C. Ethylene response pathway modulates attractiveness of plant roots to soybean cyst nematode Heterodera glycines. Sci Rep. 2017;7(1):41282. https://doi.org/10.1038/srep41282.

9. Kandoth PK, Ithal N, Recknor J, Maier T, Nettleton D, Baum TJ, et al. The soybean Rhgl locus for resistance to the soybean cyst nematode Heterodera glycines regulates the expression of a large number of stress and defenserelated genes in degenerating feeding cells. Plant Physiol. 2011;155(4):196075. https://doi.org/10.1104/pp.110.167536.

10. Arif $Y$, Singh $P$, Siddiqui $H$, Bajguz A, Hayat S. Salinity induced physiological and biochemical changes in plants: an omic approach towards salt stress tolerance. Plant Physiol Biochem. 2020;156:64-77. https://doi.org/10.1016/j. plaphy.2020.08.042.

11. Rachel C, Danyel J, Jos K, Jan BJJ. Transcriptomic and metabolomic data integration. Brief Bioinform. 2015;5:891-901.

12. Zhao X, Chen S, Wang S, Shan W, Yu X. Defensive responses of tea plants (Camellia sinensis) against tea green leafhopper attack: a multi-omics study. Front Plant Sci. 2020;10:1705. https://doi.org/10.3389/fpls.2019.01705.

13. Han Y, Zhao X, Cao G, Wang Y, Li Y, Liu D, et al. Genetic characteristics of soybean resistance to $\mathrm{HG}$ type 0 and $\mathrm{HG}$ type 1.2.3.5.7 of the cyst nematode analyzed by genome-wide association mapping. BMC Genomics. 2015;16:598.

14. Klink VP, Overall CC, Alkharouf NW, Macdonald MH, Matthews BF. A timecourse comparative microarray analysis of an incompatible and compatible response by Glycine max (soybean) to Heterodera glycines (soybean cyst nematode) infection. Planta. 2007;226(6):1423-47. https://doi.org/10.1007/ s00425-007-0581-4.

15. Klink VP, Overall CC, Alkharouf NW, MacDonald MH, Matthews BF. Laser capture microdissection (LCM) and comparative microarray expression analysis of syncytial cells isolated from incompatible and compatible soybean (Glycine max) roots infected by the soybean cyst nematode (Heterodera glycines). Planta. 2007;226(6):1389-409. https://doi.org/10.1007/ s00425-007-0578-Z

16. Liu P, Luo J, Zheng Q, Chen Q, Zhai N, Xu S, et al. Integrating transcriptome and metabolome reveals molecular networks involved in genetic and environmental variation in tobacco. DNA Res. 2020;27(2):dsaa006.

17. Mazarei M, Liu W, Alahmad H, Arelli PR, Pantalone VR, Stewart CN Jr. Gene expression profiling of resistant and susceptible soybean lines infected with soybean cyst nematode. Theor Appl Genet. 2011;123(7):1193-206. https:// doi.org/10.1007/s00122-011-1659-8.

18. Vuong TD, Sonah H, Meinhardt CG, Deshmukh R, Kadam S, Nelson RL, et al. Genetic architecture of cyst nematode resistance revealed by genome-wide association study in soybean. BMC Genomics. 2015;16(1):593. https://doi org/10.1186/s12864-015-1811-y.

19. Wang Z, Tian Z. Genomics progress will facilitate molecular breeding in soybean. Sci China Life Sci. 2015;58(8):813-5. https://doi.org/10.1007/s11427015-4908-2.

20. Zhang H, Li C, Davis EL, Wang J, Griffin JD, Kofsky J, et al. Genomewide association study of resistance to soybean cyst nematode (Heterodera glycines) HG type 2.5.7 in wild soybean (Glycine soja). Front Plant Sci. 2016;7:1214

21. Zhou Z, Jiang Y, Wang Z, Gou Z, Lyu J, Li W, et al. Resequencing 302 wild and cultivated accessions identifies genes related to domestication and improvement in soybean. Nat Biotechnol. 2015;33(4):408-14. https://doi. org/10.1038/nbt.3096.

22. Shen Y, Liu J, Geng H, Zhang J, Liu Y, Zhang $H$, et al. De novo assembly of a Chinese soybean genome. Sci China Life Sci. 2018;61(8):871-84. https://doi. org/10.1007/s11427-018-9360-0.

23. Liu Y, Du H, Li P, Shen Y, Peng H, Liu S, et al. Pan-genome of wild and cultivated soybeans. Cell. 2020;182(1):162-76.e13.

24. Schmutz J, Cannon S, Schlueter J, Ma J, Mitros T, Nelson W, et al. Genome sequence of the palaeopolyploid soybean. Nature. 2010;463(7278):178-83. https://doi.org/10.1038/nature08670.

25. Klink VP, Overall CC, Alkharouf NW, Macdonald MH, Matthews BF. Microarray detection call methodology as a means to identify and compare transcripts expressed within syncytial cells from soybean (Glycine max) roots undergoing resistant and susceptible reactions to the soybean cyst nematode (Heterodera glycines). J Biomed Biotechnol. 2010;2010:491217.

26. Ithal N, Recknor J, Nettleton D, Maier T, Baum TJ, Mitchum MG. Developmental transcript profiling of cyst nematode feeding cells in soybean roots. Mol Plant-Microbe Interact. 2007;20(5):510-25. https://doi. org/10.1094/MPMI-20-5-0510.

27. Pristner $M$, Warth B. Drug-exposome interactions: the next frontier in precision medicine. Trends Pharmacol Sci. 2020;41(12):994-1005. https://doi. org/10.1016/j.tips.2020.09.012.

28. Liu H, Brettell LE, Qiu Z, Singh BK. Microbiome-mediated stress resistance in plants. Trends Plant Sci. 2020;25(8):733-43. https://doi.org/10.1016/j.tplants.2020.03.014.

29. Feng Z, Ding C, Li W, Wang D, Cui D. Applications of metabolomics in the research of soybean plant under abiotic stress. Food Chem. 2020;310: 125914. https://doi.org/10.1016/j.foodchem.2019.125914.

30. Castro-Moretti FR, Gentzel I, Alonso M. Metabolomics as an emerging tool for the study of plant-pathogen interactions. Metabolites. 2020; 10(2):52.

31. Kantor M, Levi A, Thies J, Guner N, Parnham S. NMR analysis reveals a wealth of metabolites in root-knot nematode resistant roots of citrullus amarus watermelon plants. J Nematol. 2018;50(2):303-16. https://doi.org/1 0.21307/jofnem-2018-030.

32. Borges DGL, Echeverria JT, De Oliveira TL, Heckler RP, De Freitas MG, Damasceno-Junior GA, et al. Discovery of potential ovicidal natural products using metabolomics. PLoS One. 2019;14(1):e0211237. https://doi.org/10.13 71/journal.pone.0211237.

33. Eloh K, Sasanelli N, Maxia A, Caboni P. Untargeted metabolomics of tomato plants after root-knot nematode infestation. J Agric Food Chem. 2016; 64(29):5963-8. https://doi.org/10.1021/acs.jafc.6b02181.

34. Afzal AJ, Natarajan A, Saini N, labal MJ, Geisler M, El Shemy HA, et al. The nematode resistance allele at the rhg1 locus alters the proteome and primary metabolism of soybean roots. Plant Physiol. 2009;151(3):1264-80. https://doi.org/10.1104/pp.109.138149.

35. Kang W, Zhu X, Wang Y, Chen L, Duan Y. Transcriptomic and metabolomic analyses reveal that bacteria promote plant defense during infection of soybean cyst nematode in soybean. BMC Plant Biol. 2018;18(1):86. https:// doi.org/10.1186/s12870-018-1302-9.

36. Anand SC, Gallo KM, Baker IA, Hartwig EE. Soybean plant introductions with resistance to races 4 or 5 of soybean cyst nematode. Crop Sci. 1988;28(3).

37. Anand SC, Wrather JA, Shumway CR. Soybean genotypes with resistance to race of soybean cyst nematode. Crop Sci. 1985;25(6).

38. Ge F, Zheng N, Zhang L, Huang W, Peng D, Liu S. Chemical mutagenesis and soybean mutants potential for identification of novel genes conferring resistance to soybean cyst nematode. J Integr Agric. 2018;17(12):2734-44. https://doi.org/10.1016/S2095-3119(18)62105-7.

39. John JB, Christiansen MN. Inhibition of linolenic acid synthesis and modification of chilling resistance in cotton seedlings. Plant Physiol. 1976; 57(2):257-9. https://doi.org/10.1104/pp.57.2.257. 
40. Torresfranklin ML, Repellin A, Huynh VB, Arcylameta A, Zuilyfodil Y, Phamthi AT. Omega-3 fatty acid desaturase (FAD3, FAD7, FAD8) gene expression and linolenic acid content in cowpea leaves submitted to drought and after rehydration. Environ Exp Bot. 2009;65(2-3):162-9. https://doi.org/10.1016/j. envexpbot.2008.12.010.

41. Willemot C, Pelletier L. Influence of light and temperature on the linolenic acid content and frost resistance of winter wheat. Can J Plant Sci. 1980; 60(2):349-55.

42. Amruthesh KN, Geetha NP, Jørgensen HJL, Neergaard ED, Shetty HS. Unsaturated fatty acids from zoospores of Sclerospora graminicola induce resistance in pearl millet. Eur J Plant Pathol. 2005;111(2):125-37. https://doi org/10.1007/s10658-004-1590-9.

43. Clay WF, Bartkowski EJ, Katterman FRH. Nuclear deoxyribonucleic acid metabolism and membrane fatty acid content related to chilling resistance in germinating cotton (Gossypium barbadense). Physiol Plant. 2006;38(3): 171-5.

44. Epstein FH. The L-arginine-nitric oxide pathway. New Engl J Med. 1993; 329(27):2002-12. https://doi.org/10.1056/NEJM199312303292706.

45. Moncada S, Higgs A. The L-arginine-nitric oxide pathway. New Engl J Med. 1993;329(27):2002-12. https://doi.org/10.1056/NEJM199312303292706.

46. Moncada S, Palmer RMJ, Higgs EA. Biosynthesis of nitric oxide from Larginine: a pathway for the regulation of cell function and communication. Biochem Pharmacol. 1989;38(11):1709-15. https://doi.org/10.1016/0006-2952 (89)90403-6

47. Delledonne $M, X i a ~ Y$, Dixon RA. Nitric oxide functions as a signal in plant disease resistance. Nature. 1998;394(6693):585-8. https://doi.org/1 $0.1038 / 29087$

48. Zhou B, Guo Z, Xing J, Huang B. Nitric oxide is involved in abscisic acidinduced antioxidant activities in Stylosanthes guianensis. J Exp Bot. 2005; 56(422):3223-8. https://doi.org/10.1093/jxb/eri319.

49. Ashraf $M$, Foolad MR. Roles of glycine betaine and proline in improving plant abiotic stress resistance. Environ Exp Bot. 2007;59(2):206-16. https:// doi.org/10.1016/j.envexpbot.2005.12.006

50. Szymański M, Deniziak M, Barciszewski J. The new aspects of aminoacyltRNA synthetases. Acta Biochim Pol. 2000;47(3):821-34. https://doi.org/10.1 8388/abp.2000_4000.

51. Odonoghue $P$, Lutheyschulten Z. On the evolution of structure in aminoacyl-tRNA synthetases. Microbiol Mol Biol Rev. 2003;67(4):550-73. https://doi.org/10.1128/MMBR.67.4.550-573.2003.

52. Waard MAD. Significance of $A B C$ transporters in fungicide sensitivity and resistance. J Pestic Sci. 1997;51(3):271-5. https://doi.org/10.1002/(SICI)10969063(199711)51:3<271::AID-PS642>3.0.CO;2-\#.

53. Hans $M$. ABC transporters and their role in protecting insects from pesticides and their metabolites. Adv Insect Physiol. 2014:46:1-72.

54. Valentijnbenz M. Phytosphingosine kills Candida albicans by disrupting its cell membrane. Biol Chem. 2010;391(1):65-71.

55. Castro A, Lemos C, Falcao A, Glass NL, Videira A. Increased resistance of complex I mutants to phytosphingosine-induced programmed cell death. J Biol Chem. 2008;283(28):19314-21. https://doi.org/10.1074/jbc.M802112200.

56. Andreas P. Glutamate dehydrogenase deficiency in cerebellar degenerations: clinical, biochemical and molecular genetics aspects. Can J Neurol Sci. 1993;20(S3):S109-S16. https://doi.org/10.1017/S0317167100048617.

57. Devine GJ, Harling ZK, Scarr AW, Devonshire AL. Lethal and sublethal effects of imidacloprid on nicotine-tolerant Myzus nicotianae and Myzus persicae. Pest Manag Sci. 1996;48(1):57-62. https://doi.org/10.1002/(SICI)1096-9063 (199609)48:1<57::AID-PS435>3.0.CO;2-9.

58. Huang C, Ren J, Liang Y. Studies on isolation of nicotine from tobacco and its effects on killing insects. Acta Agric Univ Jiangxiensis. 2001;23(5):151-2.

59. Oros G, Ujváry I. Botanical fungicides: natural and semisynthetic ceveratrum alkaloids. Pest Manag Sci. 1999;55(3):253-64. https://doi.org/10.1002/(SICI)1 096-9063(199903)55:3<253::AID-PS926>3.0.CO;2-6.

60. Vänninen I, Rönnqvist M, Dahlqvist M, Forsström J. As an invasive pest in finnish tomato crops: attempt to eradicate the bugs with nicotine-based programmes. lobc/wprs Bulletin. 2011;68:185-8.

61. Zhang $H$, Wang Z, Cui G, Lin T. Difference in seedlings ammonium assimilation of wheat cultivars with different drought resistance under osmotic stress. Chin J Appl Ecol. 2009;20(10):2406-10.

62. Xu S, Hu C, Hussain S, Tan Q, Wu S, Sun X. Metabolomics analysis reveals potential mechanisms of tolerance to excess molybdenum in soybean seedlings. Ecotoxicol Environ Saf. 2018;164:589-96. https://doi.org/10.1016/j. ecoenv.2018.08.062.
63. Brown PD, Morra MJ. Control of soil-borne plant pests using glucosinolatecontaining plants. Adv Agron. 1997;61:161-231.

64. Koroleva OA, Gibson TM, Cramer R, Stain C. Glucosinolate-accumulating Scells in Arabidopsis leaves and flower stalks undergo programmed cell death at early stages of differentiation. Plant J. 2010;64(3):456-69. https:// doi.org/10.1111/j.1365-313X.2010.04339.x.

65. Arfaoui A. Effect of rhizobium isolates on isoflavonoid levels in chickpea plants infected with Fusarium oxysporum f.sp. ciceris. Phytopathol Mediterr. 2006;45(1):24-34.

66. Moustafa EM. Leaf curl virus and the flavonoid content of resistant and susceptible strains of cotton. Nature. 1961;191(4786):415.

67. Hewezi T, Baum TJ. Manipulation of plant cells by cyst and root-knot nematode effectors. Mol Plant-Microbe Interact. 2013;26(1):9-16. https://doi. org/10.1094/MPMI-05-12-0106-FI.

68. Siddique S, Grundler FM. Parasitic nematodes manipulate plant development to establish feeding sites. Curr Opin Microbiol. 2018;46:102-8. https://doi.org/10.1016/j.mib.2018.09.004.

69. Fu H, Guo X, Zhang Y, Song J, Zheng Q, Liu P, Lu P, Chen Q, Yu Y, She Y. AntDAS: automatic data analysis strategy for UPLC-QTOF-based nontargeted metabolic profiling analysis. Anal Chem. 2017;89(20):11083-90.

70. Liu P, Zhou H, Zheng Q, Lu P, Yu Y, Cao P, et al. An automatic UPLC-HRMS data analysis platform for plant metabolomics. Plant Biotechnol J. 2019; 17(11):2038-40. https://doi.org/10.1111/pbi.13180.

71. Douillet P. Disinfection of rotifer cysts leading to bacteria-free populations. J Exp Mar Biol Ecol. 1998;224(2):183-92. https://doi.org/10.1016/S0022-0981 (97)00200-1.

72. Chen X, Li S, Zhao X, Zhu X, Duan Y. Modulation of (homo) glutathione metabolism and $\mathrm{H}_{2} \mathrm{O}_{2}$ accumulation during soybean cyst nematode infections in susceptible and resistant soybean cultivars. Int J Mol Sci. 2020; 21(2):388. https://doi.org/10.3390/ijms21020388.

73. Peng H, Cui J, Long H, Huang W, Kong L, Liu S, et al. Novel pectate lyase genes of Heterodera glycines play key roles in the early stage of parasitism. PLoS One. 2016;11(3):e0149959. https://doi.org/10.1371/journa I.pone.0149959.

74. Wang G, Peng D, Gao B, Huang W, Kong L, Long H, et al. Comparative transcriptome analysis of two races of Heterodera glycines at different developmental stages. PLoS One. 2014;9(3):e91634. https://doi.org/10.1371/ journal.pone.0091634.

75. Tefft PM, Bone LW. Zinc-mediated hatching of eggs of soybean cyst nematode, Heterodera glycines. J Chem Ecol. 1984;10(2):361-72. https://doi. org/10.1007/BF00987863.

76. Lopeznicora HD, Craig JP, Gao X, Lambert KN, Niblack TL. Evaluation of cultivar resistance to soybean cyst nematode with a quantitative polymerase chain reaction assay. Plant Dis. 2012;96(10):1556-63. https://doi. org/10.1094/PDIS-12-11-1083-RE.

77. Bybd DWJ, Kirkpatrick T, Barker KR. An improved technique for clearing and staining plant tissues for detection of nematodes. J Nematol. 1983; 15(1):142-3.

78. Kong L, Wu D, Huang W, Peng H, Wang G, Cui J, et al. Large-scale identification of wheat genes resistant to cereal cyst nematode Heterodera avenae using comparative transcriptomic analysis. BMC Genomics. 2015; 16(1):1-18.

79. Kong L, Wu D, Cui J, Huang W, Peng H, Peng D. Testing and modelling the potential of three diploid plants in poaceae as a new pathosystem to investigate the interactions between cereal hosts and cereal cyst nematode (Heterodera avenae). Plant Pathol. 2016;65(4):682-8. https:/doi.org/10.1111/ppa.12427.

80. Hewezi T, Howe P, Maier T, Hussey R, Mitchum M, Davis E, et al. Cellulose binding protein from the parasitic nematode Heterodera schachtii interacts with Arabidopsis pectin methylesterase: cooperative cell wall modification during parasitism. Plant Cell. 2008;20(11):3080-93. https://doi.org/10.1105/ tpc.108.063065.

81. Riggs RD, Schmitt DP. Complete characterization of the race scheme for Heterodera glycines. J Nematol. 1988;20(3):392-5.

82. Niblack TL, Arelli PR, Noel GR, Opperman CH, Orf JH, Schmitt DP, et al. A revised classification scheme for genetically diverse populations of Heterodera glycines. J Nematol. 2002;34(4):279-88.

83. Zeng C, Lin H, Liu Z, Liu Z. Metabolomics analysis of Camellia sinensis with respect to harvesting time. Food Res Int. 2020;128:108814. https://doi.org/1 0.1016/j.foodres.2019.108814

84. Mcvey C, Mcgrath TF, Haughey SA, Elliott CT. A rapid food chain approach for authenticity screening: the development, validation and transferability of 
a chemometric model using two handheld near infrared spectroscopy (NIRS) devices. Talanta. 2020;222(2021):121533.

85. Chen Q, Li M, Wang C, Li Z, Xu J, Zheng Q, et al. Combining targeted metabolites analysis and transcriptomics to reveal chemical composition difference and underlying transcriptional regulation in Maca (Lepidium meyenii Walp.) ecotypes. Genes (Basel). 2018;9(7):335-(1).

86. Lou Q, Liu Y, Qi Y, Jiao S, Tian F, Jiang L, et al. Transcriptome sequencing and metabolite analysis reveals the role of delphinidin metabolism in flower colour in grape hyacinth. J Exp Bot. 2014;65(12):3157-64. https://doi.org/10.1 093/jxb/eru168.

87. Love MI, Huber W, Anders S. Moderated estimation of fold change and dispersion for RNA-seq data with DESeq2. Genome Biol. 2014;15(12):550. https://doi.org/10.1186/s13059-014-0550-8.

88. Conesa A, Götz S, García-Gómez JM, Terol J, Talón M, Robles M. Blast2GO: a universal tool for annotation, visualization and analysis in functional genomics research. Bioinformatics. 2005;21 (18):3674-6. https://doi.org/10.1 093/bioinformatics/bti610.

89. Kyoungwon C, Kwang-Soo C, Hwang-Bae S, Jin HI, Su-Young H, Hyerim L, et al. Network analysis of the metabolome and transcriptome reveals novel regulation of potato pigmentation. J Exp Bot. 2016;5:1519.

\section{Publisher's Note}

Springer Nature remains neutral with regard to jurisdictional claims in published maps and institutional affiliations.

Ready to submit your research? Choose BMC and benefit from:

- fast, convenient online submission

- thorough peer review by experienced researchers in your field

- rapid publication on acceptance

- support for research data, including large and complex data types

- gold Open Access which fosters wider collaboration and increased citations

- maximum visibility for your research: over $100 \mathrm{M}$ website views per year

At BMC, research is always in progress.

Learn more biomedcentral.com/submissions 